ANN A LES

UNIVERSITATIS MARIAE CURIE-SKŁODOWSKA

L UBLIN - P OLONIA

VOL. LXIX, NO. 1, 2015

SECTIO A

$83-90$

T. D. NARANG ${ }^{1}$ and SAHIL GUPTA ${ }^{2}$

\title{
Proximinality and co-proximinality in metric linear spaces
}

\begin{abstract}
As a counterpart to best approximation, the concept of best coapproximation was introduced in normed linear spaces by C. Franchetti and M. Furi in 1972. Subsequently, this study was taken up by many researchers. In this paper, we discuss some results on the existence and uniqueness of best approximation and best coapproximation when the underlying spaces are metric linear spaces.
\end{abstract}

A new kind of approximation, called best coapproximation was introduced in normed linear spaces by C. Franchetti and M. Furi [2] to obtain some characterizations of real Hilbert spaces among real Banach spaces. This study was further taken up by many researchers in normed linear spaces and Hilbert spaces (see e.g. [3], [4], [9]). But only a few have taken up this study in more general abstract spaces. The theory of best coapproximation is much less developed as compared to the theory of best approximation in abstract spaces. The present paper is also a step in this direction. In this paper, we discuss the existence and uniqueness results on best approximation and best coapproximation in metric linear spaces thereby generalizing the various known results. We start with a few definitions.

\footnotetext{
${ }^{1}$ The research work of the author has been supported by U.G.C., India under Emeritus Fellowship.

${ }^{2}$ The research work of the author has been supported by U.G.C., India under Senior Research Fellowship.

2010 Mathematics Subject Classification. 41A50, 41A52, 41A65, 35E10.

Key words and phrases. Best approximation, best coapproximation, proximinal set, co-proximinal set, Chebyshev set, co-Chebyshev set.
} 
Let $G$ be a non-empty subset of a metric space $(X, d)$. An element $g_{0} \in G$ is called a best approximation (best coapproximation) to $x \in X$ if

$$
d\left(x, g_{0}\right) \leq d(x, g)\left(d\left(g_{0}, g\right) \leq d(x, g)\right)
$$

for all $g \in G$. The set of all such $g_{0} \in G$ is denoted by $P_{G}(x)\left(R_{G}(x)\right)$. The set $G$ is called proximinal (co-proximinal) if $P_{G}(x)\left(R_{G}(x)\right)$ contains at least one element for every $x \in X$. If for each $x \in X, P_{G}(x)\left(R_{G}(x)\right)$ has exactly one element in $G$, then the set $G$ is called Chebyshev (coChebyshev).

If $x, y$ and $z$ are any three points in a metric space $(X, d)$ then $z$ is said to be a between point of $x$ and $y$ if

$$
d(x, z)+d(z, y)=d(x, y)
$$

A metric $d$ defined on $X$ is said to be a convex (strongly convex) metric if for each pair $x, y \in X, d$ determine at least one (exactly one) between point. The space $X$ together with a convex metric $d$ is called a convex metric space.

A linear space $X$ with a translation invariant metric $d$ (i.e. $d(x+z, y+z)=$ $d(x, y)$ for all $x, y, z \in X)$ such that addition and scalar multiplication are continuous on $(X, d)$ is called a metric linear space.

The space $X$ of all entire functions, i.e.

$$
X=\left\{f: f(z)=\sum_{n=0}^{\infty} a_{n} z^{n},\left|a_{n}\right|^{\frac{1}{n}} \rightarrow 0 \text { as } n \rightarrow \infty\right\}
$$

with the metric $d$ defined by

$$
d(f, g)=\max \left\{\left|a_{0}-b_{0}\right|,\left|a_{n}-b_{n}\right|^{\frac{1}{n}}, n \geq 1\right\},
$$

where $f(z)=\sum_{n=0}^{\infty} a_{n} z^{n}, g(z)=\sum_{n=0}^{\infty} b_{n} z^{n}$, is a non-normable metric linear space (see [10], p. 238).

Remarks.

(i) A proximinal (co-proximinal) subset of a metric space is closed.

(ii) Every singleton subset of a metric space is Chebyshev (co-Chebyshev).

(iii) Every closed interval in $\mathbb{R}$ is proximinal (co-proximinal).

(iv) $P_{G}\left(P_{G}(x)\right)=P_{G}(x)$ and $R_{G}\left(R_{G}(x)\right)=R_{G}(x)$.

(v) $P_{G}(x)=\left\{g_{0} \in G: d\left(x, g_{0}\right) \leq d(x, g)\right.$ for every $\left.g \in G\right\}$

$$
=G \cap B(x, d(x, G)) \text {. }
$$

(vi) $R_{G}(x)=\left\{g_{0} \in G: d\left(g_{0}, g\right) \leq d(x, g)\right.$ for every $\left.g \in G\right\}$

$$
=G \cap[\cap\{B(g, d(x, g)\}: g \in G\}] .
$$

(vii) If $G$ is a linear subspace of a metric linear space $(X, d)$ then $P_{G}^{-1}(0) \cap$ $G=\{0\}$ and $R_{G}^{-1}(0) \cap G=\{0\}$, where $P_{G}^{-1}(0)=\left\{x \in X: 0 \in P_{G}(x)\right\}$ and $R_{G}^{-1}(0)=\left\{x \in X: 0 \in R_{G}(x)\right\}$.

(viii) If $G$ is a linear subspace of a metric linear space $(X, d)$, then $d\left(g, R_{G}^{-1}(0)\right)$ $=d(g, 0)$ for every $g \in G$. 
(ix) If $G$ is a linear subspace of a metric linear space $(X, d)$, then $g_{0} \in$ $P_{G}(x)\left(R_{G}(x)\right)$ if and only if $x-g_{0} \in P_{G}^{-1}(0)\left(R_{G}^{-1}(0)\right)$.

Let $(X, d)$ be a metric linear space and $x, y \in X$, then we say that $x$ is orthogonal to $y, x \perp y$ if $d(x, 0) \leq d(x, \alpha y)$ for every scalar $\alpha$. We say that $G \perp x$ if $g \perp x$ for every $g \in G$.

Concerning orthogonality and best coapproximation, we have

Theorem 1. Let $G$ be a linear subspace of a metric linear space $(X, d)$ such that $G \perp\left(x-g_{0}\right)$, then $g_{0} \in R_{G}(x)$.

Proof. Since $G \perp\left(x-g_{0}\right)$, we have $g \perp\left(x-g_{0}\right)$ for every $g \in G$ i.e. $d(g, 0) \leq$ $d\left(g, \alpha\left(x-g_{0}\right)\right)$ for every scalar $\alpha$. Take $\alpha=1$, we get $d(g, 0) \leq d\left(g, x-g_{0}\right)$ for every $g \in G$. This gives $d\left(g_{0}, g+g_{0}\right) \leq d\left(x, g+g_{0}\right)$ for every $g \in G$. Hence $g_{0} \in R_{G}(x)$.

Note. The converse of the above theorem is also true in normed linear spaces (see [2]). We do not know whether this is true in metric linear spaces. However, in metric linear spaces, we have

Theorem 2. Let $G$ be a linear subspace of a metric linear space $(X, d)$ and $g_{0} \in G$. Then $\alpha g_{0} \in R_{G}(\alpha x)$ for every scalar $\alpha$ if and only if $G \perp\left(x-g_{0}\right)$.

Proof. Let $\alpha g_{0} \in R_{G}(\alpha x)$, then $d\left(\alpha g_{0}, g\right) \leq d(\alpha x, g)$ for every $g \in G$ i.e. $d\left(0, g-\alpha g_{0}\right) \leq d\left(\alpha x-\alpha g_{0}, g-\alpha g_{0}\right)$ for every $g \in G$. This gives $d\left(0, g^{\prime}\right) \leq d\left(\alpha x-\alpha g_{0}, g^{\prime}\right)$ for every $g^{\prime} \in G$ i.e. $d\left(g^{\prime}, 0\right) \leq d\left(g^{\prime}, \alpha x-\alpha g_{0}\right)$ for every scalar $\alpha$. Hence $G \perp\left(x-g_{0}\right)$.

Conversely, assume $G \perp\left(x-g_{0}\right)$ i.e. $g^{\prime} \perp\left(x-g_{0}\right)$ for every $g^{\prime} \in G$. This implies that $d\left(g^{\prime}, 0\right) \leq d\left(g^{\prime}, \alpha\left(x-g_{0}\right)\right)$ for every scalar $\alpha$ and for every $g^{\prime} \in G$. This gives $d\left(\alpha g_{0}, g^{\prime}+\alpha g_{0}\right) \leq d\left(\alpha x, g^{\prime}+\alpha g_{0}\right)$ for every $g^{\prime} \in G$ i.e. $d\left(\alpha g_{0}, g\right) \leq d(\alpha x, g)$ for every $g \in G$. Hence $\alpha g_{0} \in R_{G}(\alpha x)$.

Before proving the next theorem, we prove the following lemmas.

Lemma 1. Let $G$ be a closed linear subspace of a metric linear space $(X, d)$. If $x \notin G$ is such that $\alpha x$ has a best coapproximation in $G$ for every scalar $\alpha$, then every element of the subspace $\{x, G\}$ (the subspace generated by $\{x\} \cup G)$ has a best coapproximation in $G$.

Proof. Let $\alpha x+g^{\prime} \in\{x, G\}$ and $g_{0} \in R_{G}(\alpha x)$ i.e.

$$
d\left(g_{0}, g\right) \leq d(\alpha x, g) \text { for every } g \in G \text {. }
$$

This implies

$$
\left.d\left(g_{0}+g^{\prime}, g+g^{\prime}\right) \leq d\left(\alpha x+g^{\prime}, g+g^{\prime}\right)\right) \text { for every } g \in G
$$

i.e.

$$
d\left(g_{0}+g^{\prime}, g^{\prime \prime}\right) \leq d\left(\alpha x+g^{\prime}, g^{\prime \prime}\right) \text { for every } g^{\prime \prime} \in G
$$

and so $g_{0}+g^{\prime} \in R_{G}\left(\alpha x+g^{\prime}\right)$. Hence every element of the subspace $\{x, G\}$ has a best coapproximation in $G$. 
Lemma 2. If $G$ and $H$ are two subspaces of a metric linear space $(X, d)$ such that $G \subseteq H$. If $x \notin H$ has a best coapproximation in $H$ and if every element of $H$ has a best coapproximation in $G$, then $x$ has a best coapproximation in $G$.

Proof. Let $x \notin H$ be such that $h_{0} \in R_{H}(x)$, i.e. $d\left(h_{0}, h\right) \leq d(x, h)$ for every $h \in H$. Now, for $h_{0} \in H$, let $g_{0} \in G$ be such that $d\left(g_{0}, g\right) \leq d\left(h_{0}, g\right)$ for every $g \in G$. Then $d\left(g_{0}, g\right) \leq d(x, g)$ for every $g \in G$, as $G \subseteq H$. Hence $g_{0} \in R_{G}(x)$.

Theorem 3. If for every subspace $G$ of a metric linear space $(X, d)$, there exists at least one element $x \in X \backslash G$ such that $\alpha x$ has a best coapproximation in $G$ for every scalar $\alpha$, then for any subspace $G$ of $X$ every element of $X$ has a best coapproximation in $G$.

Proof. Using Lemmas 1 and 2 and proceeding as in Theorem 4.1 [5], we obtain the result.

For normed linear spaces, the following result was proved in [3].

Theorem 4. Let $G$ be a proximinal subspace of a metric linear space $(X, d)$. If $P_{G}^{-1}(0)$ is a convex set, then $G$ is Chebyshev.

Proof. Suppose $x \in X$ and $g_{1}, g_{2} \in P_{G}(x)$. Since $g_{1}, g_{2} \in P_{G}(x)$, we have $x-g_{1}, x-g_{2} \in P_{G}^{-1}(0)$. Put $x-g_{1}=g^{\prime}$ and $x-g_{2}=g^{\prime \prime}$, where $g^{\prime}, g^{\prime \prime} \in P_{G}^{-1}(0)$. We first claim that $g_{1}-x \in P_{G}^{-1}(0)$. Since $0 \in P_{G}\left(x-g_{1}\right)$, we have $d\left(x-g_{1}, 0\right) \leq d\left(x-g_{1}, g\right)$ for every $g \in G$. This implies $d\left(g_{1}-\right.$ $x, 0) \leq d\left(-g, g_{1}-x\right)$ for every $g \in G$ i.e. $d\left(g_{1}-x, 0\right) \leq d\left(g_{1}-x, g^{\prime}\right)$ for every $g^{\prime} \in G$. Therefore, $g_{1}-x \in P_{G}^{-1}(0)$. This proves our claim. Now, $x-g_{2}, g_{1}-x \in P_{G}^{-1}(0)$ and $P_{G}^{-1}(0)$ is convex, we have $\frac{1}{2}\left[\left(x-g_{2}\right)+\left(g_{1}-x\right)\right] \in$ $P_{G}^{-1}(0)$ i.e. $\frac{1}{2}\left[g^{\prime \prime}-g^{\prime}\right] \in P_{G}^{-1}(0)$. Also $\frac{1}{2}\left[g^{\prime \prime}-g^{\prime}\right]=\frac{1}{2}\left[g_{1}-g_{2}\right] \in G$ and so $\frac{1}{2}\left[g_{1}-g_{2}\right] \in P_{G}^{-1}(0) \bigcap G=\{0\}$. This implies $g_{1}=g_{2}$. Hence $G$ is Chebyshev.

Remark. If we take $G$ to be a proximinal subset of a metric linear space $(X, d)$, then the convexity of $P_{G}^{-1}(0)$ need not imply the Chebyshevity of $G$.

Example 1. Let $X=\mathbb{R}$ and $G=\{0,1,2,3, \ldots, 10\}$ then $G$, being a compact set is proximinal (see [1]) and $P_{G}^{-1}(0)=(-\infty, 0.5]$ is a convex set but $G$ is not Chebyshev as $P_{G}(0.5)=\{0,1\}$.

Analogously, concerning the co-Chebyshevity of $G$, we have the following result:

Theorem 5. Let $G$ be a co-proximinal subspace of a metric linear space $(X, d)$. If $R_{G}^{-1}(0)$ is a convex set, then $G$ is co-Chebyshev.

Proof. The proof runs on similar lines as that of Theorem 4. 
Remark. If we take $G$ to be a co-proximinal subset of a metric linear space $(X, d)$, then the convexity of $R_{G}^{-1}(0)$ need not imply the co-Chebyshevity of $G$.

Example 2. Let $X=\mathbb{R}$ and $G=[0, \infty)$, then $R_{G}^{-1}(0)=(-\infty, 0]$ and $R_{G}(-1)=[0,1]$ i.e. $R_{G}^{-1}(0)$ is a convex set but $G$ is not co-Chebyshev.

Before proceeding further, we recall the following results on coapproximation proved in [8].

Lemma 3 ([8] Theorems 5 and 6). Let $G$ be a closed linear subspace of a metric linear space $(X, d)$, then the following statements are equivalent:

(i) $G$ is co-proximinal.

(ii) $X=G+R_{G}^{-1}(0)$.

(iii) For the canonical mapping $w_{G}: X \rightarrow X / G$ defined by $w_{G}(x)=x+G$, we have

$$
w_{G}\left(R_{G}^{-1}(0)\right)=X / G
$$

Lemma 4 ([8], Theorem 7). For a closed linear subspace $G$ of a metric linear space $(X, d)$, the following statements are equivalent:

(i) $G$ is co-Chebyshev.

(ii) $X=G \oplus R_{G}^{-1}(0)$, where $\oplus$ means that the sum decomposition of each $x \in X$ is unique.

(iii) $G$ is co-proximinal and $\left[R_{G}^{-1}(0)-R_{G}^{-1}(0)\right] \cap G=\{0\}$.

(iv) $G$ is co-proximinal and the restriction map $w_{G}\left(R_{G}^{-1}(0)\right)$ is one to one.

Remark. For best approximation, Lemmas 3 and 4 were proved in [7].

Concerning the proximinality of $R_{G}^{-1}(0)$, we have

Theorem 6. Let $G$ be a co-proximinal subspace of a metric linear space $(X, d)$. If $R_{G}^{-1}(0)$ is a subspace of $X$ then $R_{G}^{-1}(0)$ is proximinal in $X$.

Proof. Since $R_{G}^{-1}(0)$ is linear subspace of the metric linear space $(X, d), G$ is co-Chebyshev in $X$, by Theorem 5. Therefore, $X=G \oplus R_{G}^{-1}(0)$ by Lemma 4 . Let $x \in X \backslash R_{G}^{-1}(0)$ be arbitrary then $x=g_{1}+g_{2}, g_{1} \in G, g_{2} \in R_{G}^{-1}(0)$. Consider $d\left(x, g_{2}\right)=d\left(x-g_{2}, 0\right)=d\left(g_{1}, 0\right)=d\left(g_{1}, R_{G}^{-1}(0)\right)$. This gives $d\left(x, g_{2}\right)=d\left(x-g_{2}, R_{G}^{-1}(0)\right)=d\left(x, R_{G}^{-1}(0)\right)$. Hence $R_{G}^{-1}(0)$ is proximinal in $X$.

Before proving the next theorem, we prove the following lemma.

Lemma 5. Let $H$ be a co-proximinal linear subspace of a metric linear space $(X, d)$, then there exists an element $z \in X \backslash\{0\}$ such that $0 \in R_{H}(z)$.

Proof. Let $x \in X \backslash H$. Since $H$ is co-proximinal, there exists $y_{0} \in R_{H}(x)$ and so $x-y_{0} \in R_{H}^{-1}(0)$. Hence $0 \in R_{H}\left(x-y_{0}\right), x-y_{0} \neq 0$. 
Theorem 7. Let $G$ be a co-proximinal linear subspace of a metric linear space $(X, d)$, then $G$ is closed and in every linear subspace $F_{x} \subset X(x \in$ $X \backslash G)$ of form $F_{x}=G \oplus[x]$ there exists an element $z \in F_{x} \backslash\{0\}$ such that $0 \in R_{G}(z)$.

Proof. Since $G$ is co-proximinal, $G$ is closed and $G$ is co-proximinal in every subspace $F_{x} \subset X(x \in X \backslash G)$ of form $F_{x}=G \oplus[x]$. Then by using the above lemma, there exists $z \in F_{x} \backslash\{0\}$ such that $0 \in R_{G}(z)$.

Note. A similar result for proximinality was proved in [11] for normed linear spaces.

For the metric coprojection $R_{G}: X \rightarrow 2^{G}$, the graph of $R_{G}$ is denoted by $G\left(R_{G}\right)$ i.e. $G\left(R_{G}\right)=\left\{\left(x, R_{G}(x)\right): x \in X\right\}$. Concerning the graph of $R_{G}$, we have the following theorem (a similar result for the metric projection $P_{G}$ was proved in $[6])$.

Theorem 8. If $G$ is a co-Chebyshev subset of a metric space $(X, d)$ then the graph of the metric coprojection $R_{G}$ is closed.

Proof. Let $(y, z)$ be a limit point of $G\left(R_{G}\right)=\left\{\left(x, R_{G}(x)\right): x \in X\right\}$. Then there exists a sequence $\left(y_{n}, R_{G}\left(y_{n}\right)\right)$ in $G\left(R_{G}\right)$ such that $\left(y_{n}, R_{G}\left(y_{n}\right)\right) \rightarrow$ $(y, z)$ i.e. $y_{n} \rightarrow y, R_{G}\left(y_{n}\right) \rightarrow z$. Since $d\left(R_{G}\left(y_{n}\right), g\right) \leq d\left(y_{n}, g\right)$ for every $g \in G$, we get $d(z, g) \leq d(y, g)$ for every $g \in G$ and so $z \in R_{G}(y)$. Since $G$ is co-Chebyshev, $\{z\}=R_{G}(y)$. Therefore $(y, z) \in G\left(R_{G}\right)$ and hence $G\left(R_{G}\right)$ is closed.

\section{Remarks.}

(1) A proximinal (co-proximinal) subset of a metric space is closed but the converse is not true.

Example 3. Let $X=\mathbb{R}-\{p\}$, then $M=(p, p+1]$ is a closed subset of the metric space $X$ with usual metric but it is not proximinal.

Example 4. The set of natural numbers is a closed subset of the real line $\mathbb{R}$ but it is not co-proximinal.

(2) Whereas a compact subset of a metric space is proximinal (see [1]), it need not be co-proximinal.

Example 5. Let $X=\mathbb{R}^{2}$ and $M=\left\{(x, y) \in \mathbb{R}^{2}: x^{2}+y^{2}=1\right\}$, then $M$ is a compact subset of $\mathbb{R}^{2}$ and hence proximinal. However, $M$ is not co-proximinal as $(0,0) \in \mathbb{R}^{2}$ does not have any best coapproximation in $M$.

This example also shows that a proximinal subset of a metric space need not be co-proximinal.

(3) A co-proximinal subset of a metric space need not be proximinal.

Example 6. Let $X=\mathbb{R}-\{1\}$ and $M=(1,2]$, then $M$ a is co-proximinal subset of $X$ but it is not proximinal. 
(4) A Chebyshev subset of a metric space need not be co-Chebyshev.

Example 7. Let $X=\mathbb{R}$ and $G=[1,2]$, then $G$ is Chebyshev in the real line $\mathbb{R}$ but it is not co-Chebyshev.

(5) A co-Chebyshev subset of a metric space need not be Chebyshev.

Example 8. Let $X=R^{2}$ with the metric $d\left(\left(x_{1}, y_{1}\right),\left(x_{2}, y_{2}\right)\right)=\left|x_{1}-x_{2}\right|+$ $\left|y_{1}-y_{2}\right|$ and $G=\left\{(x, y) \in R^{2}: x=y\right\}$. Then, $X$ is a real Banach space and $G$ is a proximinal subspace of $X$. We have $P_{G}(x, y)=\{\alpha(x, x)+$ $(1-\alpha)(y, y): 0 \leq \alpha \leq 1\}$ and $R_{G}(x, y)=\left\{\left(\frac{x+y}{2}, \frac{x+y}{2}\right)\right\}$. Hence $G$ is co-Chebyshev but not Chebyshev.

(6) In a Hilbert space $H$, it is known (see [2]) that if $M$ is a subspace of $H$, then the set of best approximations and set of best coapproximations are the same. But if we take $M$ to be a subset of $H$, then this need not be so.

Example 9. Let $X=\mathbb{R}^{2}$ and $M=\left\{(x, y) \in \mathbb{R}^{2}: x^{2}+y^{2}=1\right\}$, then every element of $M$ is best approximation to $(0,0)$, but $(0,0)$ does not have any best coapproximation in $M$.

(7) It is known (see [6]) that if $G$ is a subset of a convex metric space $(X, d)$ and $x \in P_{G}^{-1}\left(g_{0}\right)=\left\{x_{0} \in X: d\left(x_{0}, g_{0}\right)=d\left(x_{0}, G\right)\right\}$, then $z \in P_{G}^{-1}\left(g_{0}\right)$ for every $z$ between $x$ and $g_{0}$. But such a result is not true in case of best coapproximation.

Example 10. Let $X=\mathbb{R}$ and $G=(-1,1)$ then $-2 \in R_{G}^{-1}(0)$, but $-1 \notin$ $R_{G}^{-1}(0)$.

(8) It is known (see [6]) that if $G$ is a Chebyshev subset of a convex metric space $(X, d)$, then $P_{G}(z)=P_{G}(x)$, where $z \in X$ is any element between $x$ and $P_{G}(x)$. Does such a result hold for best coapproximation?

Acknowledgement. The authors are thankful to the learned referee for valuable comments and suggestions leading to an improvement of the paper.

\section{REFERENCES}

[1] Cheney, E. W., Introduction to Approximation Theory, McGraw Hill, New York, 1966.

[2] Franchetti, C., Furi, M., Some characteristic properties of real Hilbert spaces, Rev. Roumaine Math. Pures Appl. 17 (1972), 1045-1048.

[3] Mazaheri, H., Maalek Ghaini, F. M., Quasi-orthogonality of the best approximant sets, Nonlinear Anal. 65 (2006), 534-537.

[4] Mazaheri, H., Modaress, S. M. S., Some results concerning proximinality and coproximinality, Nonlinear Anal. 62 (2005), 1123-1126.

[5] Muthukumar, S., A note on best and best simultaneous approximation, Indian J. Pure Appl. Math. 11 (1980), 715-719.

[6] Narang, T. D., Best approximation in metric spaces, Publ. Sec. Mat. Univ. Autonoma Barcelona 27 (1983), 71-80. 
[7] Narang, T. D., Best approximation in metric linear spaces, Math. Today 5 (1987), $21-28$.

[8] Narang, T. D., Singh, S. P., Best coapproximation in metric linear spaces, Tamkang J. Math. 30 (1999), 241-252.

[9] Papini, P. L., Singer, I., Best coapproximation in normed linear spaces, Monatsh. Math. 88 (1979), 27-44.

[10] Rao, K. Chandrasekhara, Functional Analysis, Narosa Publishing House, New Delhi, 2002 .

[11] Singer, I., Best Approximation in Normed Linear Spaces by Elements of Linear Subspaces, Springer-Verlag, New York, 1970.

T. D. Narang

Department of Mathematics

Guru Nanak Dev University

Amritsar-143005

India

e-mail: tdnarang1948@yahoo.co.in

Received July 25, 2014
Sahil Gupta

Department of Mathematics

Guru Nanak Dev University

Amritsar-143005

India

e-mail: sahilmath@yahoo.in 